\title{
Interfacial Flows of Contact Line Dynamics and Liquid Displacement in a Circular Microchannel
}

\author{
Changfei Yan, Huihe Qiu \\ Department of Mechanical and Aerospace Engineering, the Hong Kong University of Science and Technology \\ Clear Water Bay, Kowloon, Hong Kong \\ changfei.yan@connect.ust.hk; meqiu@ust.hk
}

\begin{abstract}
In this paper, we report experimental and analytical studies of the interfacial flows of contact line dynamics and liquid film displacement of gas-liquid two-phase flows in a capillary tube. To quantitatively measure and analyse the interfacial liquid film displacement behaviour, an optical technique for measuring the interfacial liquid film between the liquid and gas in a capillary tube has been developed. The interfacial film dynamics is detected by a very sensitive laser spatial fringe projection technique and a high speed camera. The spatial frequencies of the fringe patterns are calculated utilizing image processing technique which are used to determine the interfacial film thickness between the gas plug and the channel wall. The uncertainty of the measurements is less than several hundred nanometres. Because the resolution of the film measurements is very high, it is possible to determine whether a liquid film exists or not for different flow capillary numbers, therefore, the critical capillary numbers can be determined. Pressure-driven flow model is used to analyse the interfacial evolution and the contact line motion. It is found that the critical capillary number is affected by the surface tension of liquids and wall surface wettability. The newly developed analytical model is validated by experimental results utilizing the interfacial film measurement technique.
\end{abstract}

Keywords: Interfacial Film Displacement, Critical Capillary Number, Dewetting, Contact Line Dynamics

\section{Introduction}

In the last two decade, Microfluidics develops quite rapidly in plenty of areas, such as chemical synthesis [1], drug screening [2], emulsification [3], reactors [4], oscillation heat pipes [5] and biomedical diagnostics [6]. The thin liquid film generated from condensation and liquid motion will transform to vapour, during which process more heat can be transferred from one side to another comparing with conduction and normal convection heat transfer.

Understanding interfacial film displacement and contact line dynamics in liquid-gas plug flow is important in twophase flow research. The dynamics of interfacial film displacement are often controlled by the capillary number, and usually include events of how the liquid-gas interface evolves, depending on flow conditions and fluid properties [7-12]. However, the processes of liquid film formation, development and breakup are complex which involve the interfacial tension between fluid-fluid and fluid-solid, and the capillary induced instability of thin liquid film breakup. The surface adhesion between the liquid and solid can also play important role during the liquid displacement. As a consequence of the small length scale, the capillary forces play a fundamental role in the physics of the phenomena. In this paper, we present a new phenomenon under water-air plug flow in a capillary tube. Although liquid-gas plug flows have been extensively studied, they typically studied the interfacial film dynamics under different flow conditions without taking the wall wettability into consideration. This might be correct in macroscale. However, this may not be correct when the capillary diameter is small where surface wetting become dominant. In that case, the interfacial film dynamic and breakup are affected by the wall adhesion. In this paper, we report experiments and simulations of the interfacial flows of contact line dynamics and liquid film displacement of gas-liquid two-phase flows in a circular microchannel.

\section{Methodology}

To quantitatively analyse the interfacial liquid film displacement behaviour, an optical technique for measuring the interfacial liquid film between the liquid and gas in a circular microchannel is developed recently [13]. The interfacial film dynamics is detected by a very sensitive laser spatial fringe projection technique and a high speed camera. The spatial 
frequencies of the fringe patterns are calculated utilizing image processing technique which are used to determine the interfacial film thickness between the gas plug and the channel wall. The interfacial film dynamics can be predicted utilizing the measured film thicknesses under various capillary numbers. Because the resolution of the film measurements is very high, it is possible to determine whether a liquid film exist or not when the flow capillary number is changed. Therefore, it is possible to determine the critical capillary numbers that governing the interfacial fluid dynamics for different wall wettability. We derived the dynamic dewetting conditions and a critical capillary number was found that determines the film breakup or lubrication. A constant effective pressure force is applied on interfacial fluids to simulate a pressure-driven flow, and an interface model is used to analyse the interfacial evolution and the contact line motion. It is found that the critical capillary number is affected by the surface tension of liquids and wall surface wettabilities. The newly developed analytical model is validated by experimental results utilizing the interfacial film measurement technique, which demonstrates that the critical capillary number is associated with the contact angle and dimensionless slip length. The novel measurement technique and analytical model for the contact line dynamics and interfacial liquid displacement are described below in details.

\subsection{Experimental Setup}

To measure the interfacial liquid film thickness, laser interferometer technique was developed [16]. The scattering rays in a capillary cross-section when a laser enters a capillary are shown in Fig. 2.

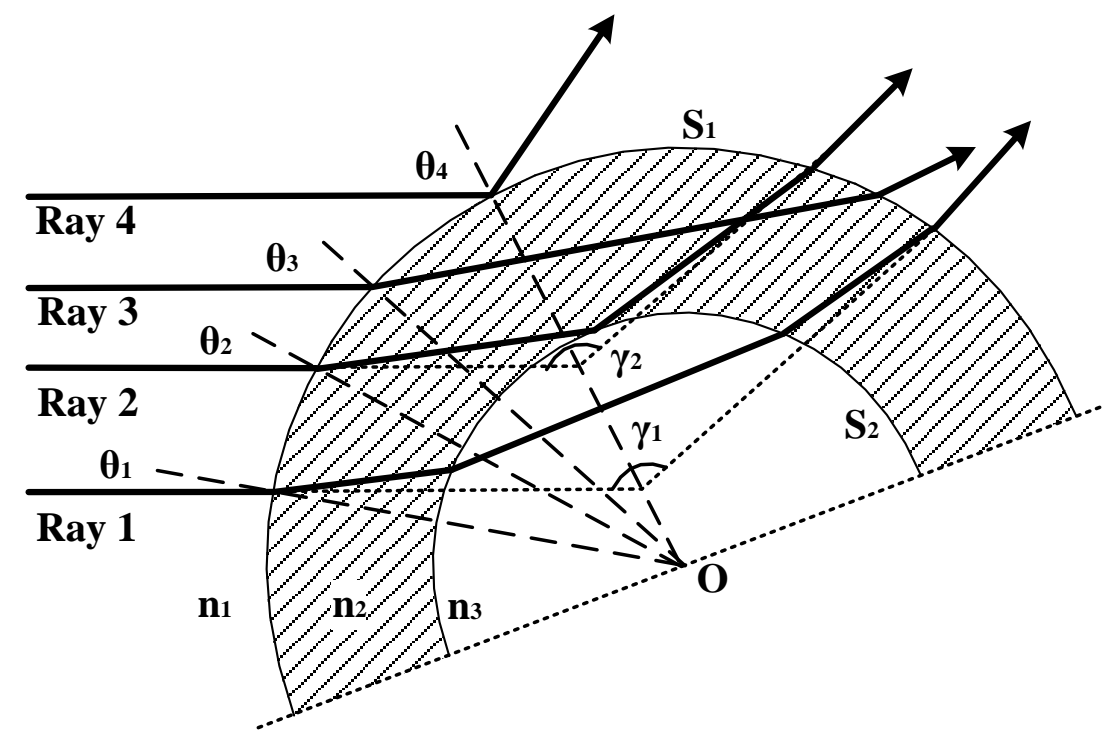

Fig. 1: The refraction and reflex cross-section when a capillary contains a gas bubble ( $\theta$ is the incident beam angle, $\gamma$ is the output beam angle and $\mathrm{n}_{1}, \mathrm{n}_{2}$, and $\mathrm{n}_{3}$ are reflective indices of glass wall, liquid film and air, respectively).

Between two surfaces S1 and S2 (S1, the interface of glass and liquid; S2, the interface of liquid and gas), there are 3 layers of media, namely capillary glass, liquid and gas. The refractive indices of the capillary tube, the liquid and the gas are $n_{1}, n_{2}$ and $n_{3}$, respectively. One assumption of this measurement method is $n_{1}>n_{2}>n_{3}$, which is normally the case for glass, liquid and air. When incident laser rays enter the surface between the capillary glass and liquid from the left, the reflected rays can be divided into 4 sets, where Ray 1 has the propagating path of S1-S2-S2-S1, the propagating path of Ray 2, is S1-S2-S1, Ray 3 has the propagating path of S1-S1 and Ray 4 has the propagating path of S1-S1. Rays acting like Ray 1 are defined as a punctured set and those of Ray 2 as a reflected set. Considering the incident beam angle $(\theta)$, the output beam angle $(\gamma)$ and the radius of the gas bubble $(k R)$, the annular liquid film thickness can be calculated as $h=(1-k) R$. The liquid film thickness $\mathrm{h}$ can be calculated by: 


$$
\left\{\begin{array}{c}
\frac{\pi}{2}-\operatorname{asin}\left(\frac{n_{1}}{n_{3}} \sin \theta_{e 1}\right)=\theta_{e 2}-\theta_{e 1} \\
\frac{\pi}{2}-\operatorname{asin}\left(\frac{n_{1}}{\left(1-\frac{h}{R}\right) n_{3}} \sin \theta_{1}\right)=\theta_{2}-\theta_{1}-\left[\operatorname{asin}\left(\frac{n_{1}}{n_{2}} \sin \theta_{2}\right)-\operatorname{asin}\left(\frac{n_{1}}{n_{2}} \sin \theta_{1}\right)\right] \\
-\left[\operatorname{asin}\left(\frac{n_{1}}{\left(1-\frac{h}{R}\right) n_{2}} \sin \theta_{1}\right)-\operatorname{asin}\left(\frac{n_{1}}{\left(1-\frac{h}{R}\right) n_{2}} \sin \theta_{2}\right)\right] \\
\theta_{e 2}=\theta_{2}+\operatorname{asin}\left(\frac{\sin \theta_{2}^{\prime}}{\left.1-\frac{h}{R}\right)-\theta_{2}^{\prime}}\right. \\
\frac{f_{e}}{f_{2}}=\frac{\sin \theta_{e 1}-\sin \theta_{e 2}}{\sin \theta_{1}-\sin \theta_{2}}
\end{array}\right.
$$

Where $\theta_{1}^{\prime}=\frac{n_{1}}{n_{2}} \sin \theta_{1}$ and $\theta_{2}^{\prime}=\frac{n_{1}}{n_{2}} \sin \theta_{2} ; f$ is the frequency of interference fringes on the CCD, the subscript $e$ is for empty capillary. In practice, after the experiment has been set up, the spatial frequency of fringe patterns of an empty capillary is recorded for reference before a measurement is conducted. The uncertainty of the measurements is less than several hundred nanometres in the experiments.

\subsection{Analytical Modelling}

Considering a pressure driven slug bubble moving forward with speed $U$ in a circular microchannel, $u$ and $v$ are the velocity of liquid in $\mathrm{x}$ and $\mathrm{y}$ directions, as shown in Fig. 2 (a). The conservation of mass should be: $\frac{\partial u}{\partial x}+\frac{\partial v}{\partial y}=0$. The Navier-Stokes equation in liquid film could be simplified dramatically by lubrication theory if there is no evaporation occurred.

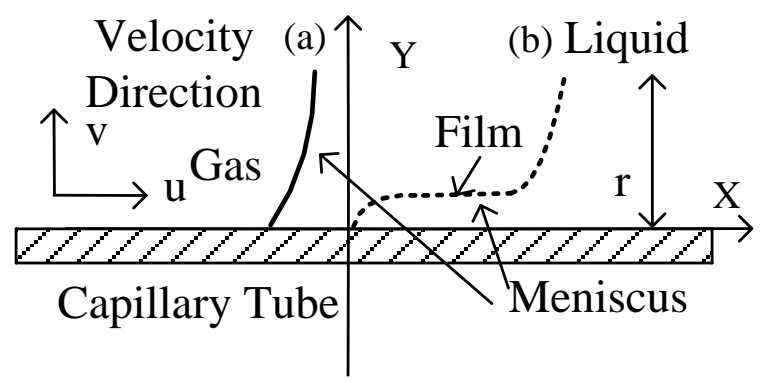

Fig. 2: Schematic of meniscus moving in a capillary: (a) with low capillary number; (b) with high capillary number.

We use the pressure item $\frac{P_{x} h}{3 \mu}$ to replace the gravity term and assume the pressure drop of air near the interface is the same with the one in infinite long position. Taking the boundary conditions into consideration, the critical capillary number can be determined in the following:

$$
\exp \left[\frac{\theta_{e}{ }^{3}}{9 C a}-\frac{\ln \left(\theta_{e}+1\right)}{\theta_{e}}+\ln \left(\frac{\theta_{e}}{\theta_{e}+1}\right)-\frac{9}{2} k \lambda_{r}^{2} \theta_{e}{ }^{2}+\frac{\ln 2}{\theta_{e}}\right]=\frac{\left(\frac{3}{2} C a\right)^{\frac{1}{3}}}{3 \lambda_{r} \pi A i\left(s_{1}\right)^{2}}
$$

where $\theta_{e}$ is the equilibrium contact angle, $\lambda_{r}$ is the dimensionless slip length, $k=\frac{\mu_{\text {air }}}{\mu_{\text {liquid }}}$ is the ratio of dynamics viscosity and $\mathrm{Ca}$ is the capillary number. 
The critical capillary numbers under different contact angels are shown in Fig 3. Although the exact slip length value in normal wettability materials is still unknown, the scale of slip length in this paper could be set as $2 \mathrm{~nm}$. The predicted critical capillary numbers using equation (2) agree well with the experimental data as shown in Fig. 3.

\section{Conclusion}

For the first time, we derived analytical solution regarding the critical capillary number with the wettability effect in a pressure driven circular microchannel. A lubrication model is utilized in the theoretical derivation, which limits the equations here on a hydrophilic surface. By matching the curvatures of the intermediate and outer regions, we can obtain an equation for predicting the critical capillary number. The critical capillary numbers calculated by this equation match well with the experiment data.

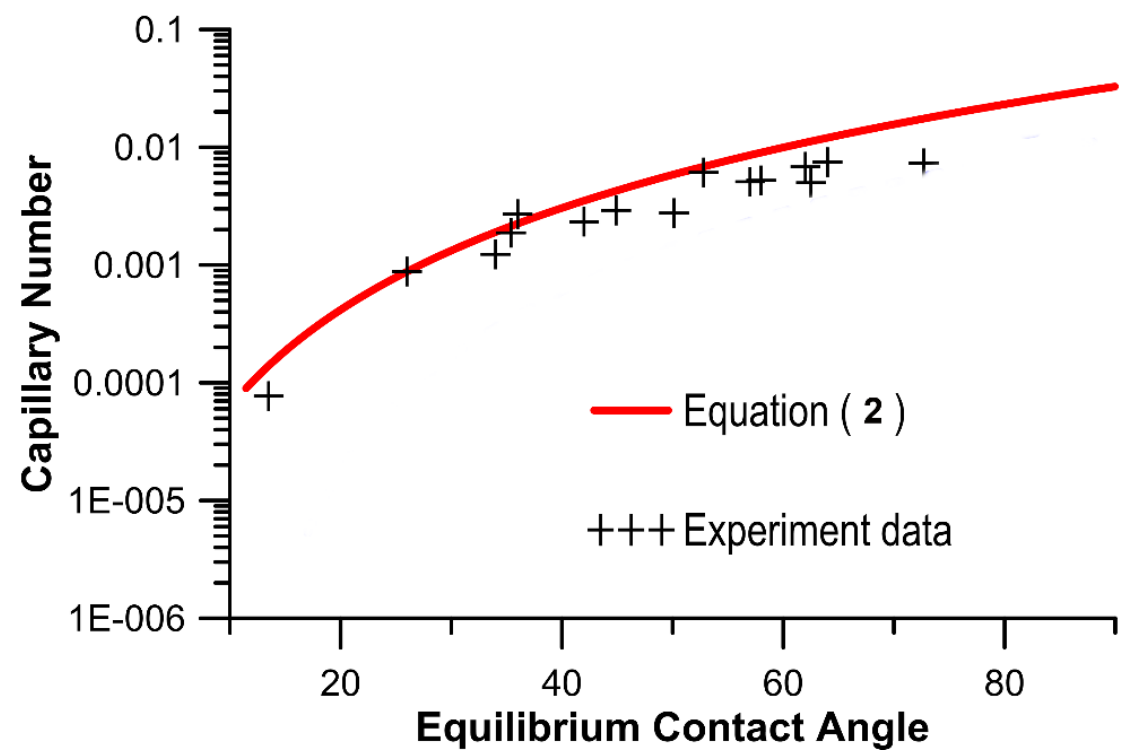

Fig. 3: The relationship of critical capillary number and equilibrium contact angle.

\section{Acknowledgements}

This work was supported by the Research Grants Council (RGC) of the Hong Kong Special Administration Region, China (Grant Nos. 617812 and 16207515).

\section{References}

[1] K. Elvira, X. Solvas, R. Wootton, A. deMello, "The past, present and potential for microfluidic reactor technology in chemical synthesis," Nature Chemistry, vol. 5, pp. 905-915, 2013.

[2] P. Dittrich, A. Manz, "Lab-on-a-chip: microfluidics in drug discovery," Nature Reviews Drug Discovery, vol. 5, pp. 210-218, 2006.

[3] P. Chen, R. Erb, A. Studart, "Designer Polymer-Based Microcapsules Made Using Microfluidics," Langmuir, vol. 28, pp. 144-152, 2012.

[4] Z. Nie, S. Xu, M. Seo, P. Lewis, E. Kumacheva, "Polymer Particles with Various Shapes and Morphologies Produced in Continuous Microfluidic Reactors," Journal of the American Chemical Society, vol. 127, pp. 80588063, 2005.

[5] H. Ma, M. Hanlon, C. Chen, "An investigation of oscillating motions in a miniature pulsating heat pipe," Microfluidics and Nanofluidics, vol. 2, pp. 171-179, 2005.

[6] R. Gorkin, J. Park, J. Siegrist, M. Amasia, B. Lee, J. Park, J. Kim, H. Kim, M. Madou, Y. Cho, "Centrifugal microfluidics for biomedical applications," Lab on a Chip, vol. 10, pp. 1758, 2010.

[7] H. Ma, C. Wilson, Q. Yu, K. Park, U. Choi, M. Tirumala, "An Experimental Investigation of Heat Transport Capability in a Nanofluid Oscillating Heat Pipe," Journal of Heat Transfer, vol. 128, pp. 1213, 2006.

[8] F. P. Bretherton, "The motion of long bubbles in tubes," Journal of Fluid Mechanics, vol. 10, 166-188, 1961. 
[9] E. J. Soares, R. L. Thompson "Flow regimes for the immiscible liquid-liquid displacement in capillary tubes with complete wetting of the displaced liquid," Journal of Fluid Mechanics, vol. 641, pp. 63-84, 2009.

[10] H.-H. Wei, D. Halpern, J. B. Grotberg, "Linear stability of a surfactant-laden annular film in a time-periodic pressure-driven flow through a capillary," Journal of Colloid and Interface Science, vol. 285, pp. 769-780, 2005.

[11] L. Hocking, "Meniscus draw-up and draining," European Journal of Applied Mathematics, vol. 12, p. $195,2001$.

[12] H. F. Ji, H. H. Qiu, "2D fringe probing of liquid film dynamics of a plug bubble in a micropipe," Measurement Science and Technology, vol. 20, p. 025402, 2009.

[13] C. Yan, H. Qiu, "Measurements of interfacial dynamics of gas-liquid displacement in a capillary," Measurement Science and Technology, vol. 27, no. 6, p. 065204, 2016. 